25.0\% $(n=9)$, and renal cell carcinoma 11.1\% $(n=4)$ respectively. The calculated lifetime prevalence (risk) of developing an EPM (Adjusted for Multiple Primaries - AMP method) in the general Maltese population is $19.5 \%$ (1 in 5). This was not found to be statistically significantly different when compared to the IPMN patient cohort $(\mathrm{p}=0.86)$.

Conclusions A previous history or synchronous histological diagnosis of EPM was not shown to be more prevalent in patients diagnosed with an IPMN of the pancreas, as compared to the general Maltese population. Given these findings, there is currently no rationale for undergoing further thorough investigations for an EPM in IPMN patients. The need for prospective, long-term follow-up studies in such patients is paramount to establish incidence rates for EPMs following an IPMN diagnosis.

\section{Small Bowel \& Nutrition}

\section{OWE-019 MECHANISMS OF CHEMOTHERAPY-INDUCED DIARRHOEA}

Stephanie French*, Andrea Davies, Munir Pirmohamed. University Of Liverpool, Liverpool, UK

\subsection{6/gutjnl-2018-BSGAbstracts.315}

Introduction Bcr-Abl inhibitors, such as bosutinib and imatinib, are predominantly used for treatment of chronic myeloid leukaemia. However, lower gastrointestinal toxicity, such as diarrhoea, is a prevalent adverse drug reaction (ADR). For example, bosutinib and imatinib cause diarrhoea in up to $90 \%$ and $50 \%$ of patients, respectively. ${ }^{1}$ This can decrease patient quality of life, treatment efficacy and in severe cases cause patient hospitalisation. We aim to elucidate the mechanism of $\mathrm{Bcr}-\mathrm{Abl}$ inhibitor-induced diarrhoea to help abrogate the aforementioned issues.

Methods Caco-2 cells (human colorectal cancer cells resembling small intestinal cells) were differentiated into monolayers of polarised enterocytes and utilised as an in vitro model. Cells were seeded into transwells and electrical resistance or flux of FITC-dextran (a fluorescently labelled polysaccharide) across the monolayer was measured to assess changes in paracellular permeability. Enteroids (small intestinal organoids) produced from male $\mathrm{BALB} / \mathrm{c}$ mice were used as an ex vivo model. Changes in permeability of enteroids were determined by leakage of injected FITC-dextran out of the enteroid. Changes in mRNA levels, protein levels and protein localization of tight junction components were studied using RTqPCR, immunoblotting and immunofluorescence, respectively. Drug-induced cell death was assessed by CellTitreGlo and Toxilight assays for Caco-2 cells and enteroids, respectively. Results were analysed by ANOVA and are representative of $\geq 3$ independent experiments.

Results $25 \mu \mathrm{M}$ bosutinib increased paracellular permeability of Caco-2 monolayers to ions and FITC-dextran (ANOVA, $\mathrm{p}<0.05$ ), whilst imatinib was less effective at inducing this change. $10 \mu \mathrm{M}$ bosutinib increased enteroid leakage (ANOVA, $\mathrm{p}<0.01$ ) but $10 \mu \mathrm{M}$ imatinib had no effect. All concentrations tested were sub-apoptotic.

In Caco-2 cells, bosutinib caused relocalization and decreased protein levels of intercellular junction proteins Ecadherin, Occludin and ZO-1. Bosutinib also transiently decreased mRNA levels of ZO-1 but not that of E-cadherin or Occludin. Imatinib did not alter mRNA levels, protein levels or localization of any of these proteins.

Endoplasmic reticulum (ER) stress is involved in intercellular junction degradation; ${ }^{2}$ therefore, we assessed whether Bcr$\mathrm{Abl}$ inhibitors could induce ER stress. However, no increase in ER stress markers BiP or CHOP were detected after bosutinib or imatinib treatment in Caco-2 cells.

Conclusions Decreased intestinal barrier integrity is likely an important factor in the aetiology of bosutinib-induced diarrhoea. This is potentially mediated by intercellular junction degradation. Understanding the mechanism by which Bcr-Abl inhibitors induce diarrhoea will aid in abrogation of diarrhoea ADRs.

\section{REFERENCES}

1. Br J Haematol. 2015;168:69.

2. Toxicol Appl Pharmacol. 2014;278:45.

\section{OWE-020 SURVIVAL AND CT DEFINED SARCOPENIA IN PATIENTS WITH INTESTINAL FAILURE ON HPN}

${ }^{1,2} \mathrm{SM}$ Oke* ${ }^{*}{ }^{1} \mathrm{~B}$ Rye, ${ }^{1,2} \mathrm{G}$ Malietzis, ${ }^{1} \mathrm{R}$ Baldwin, ${ }^{2} \mathrm{RA}$ Bottle, ${ }^{1,2} \mathrm{SM}$ Gabe, ${ }^{1} \mathrm{PFC}$ Lung. ${ }^{1} \mathrm{St}$ Mark's Hospital, Harrow, UK; ${ }^{2}$ Imperial College London, UK

\subsection{6/gutjnl-2018-BSGAbstracts.316}

Introduction Sarcopenia is recognised in patients with intestinal failure (IF) and has been associated with poorer survival in several chronic diseases. CT can measure sarcopenia through a L3 skeletal muscle index (LSMI).

We aim to evaluate the prevalence of sarcopenia in our IF population using LSMI, and evaluate the effect of home parenteral support (HPN) on LSMI and survival. Additionally, we aim to assess any association between LSMI, BMI and other anthropometric measurements.

Methods IF patients on HPN treated at St Mark's Hospital between 1/1/2006-1/10/2016 were identified from a prospectively maintained database. Patients were included if they were on HPN and had 2 CTs: the first $\leq 30$ days before start of HPN (pre-HPN); the second $\geq 100$ days from HPN start (post-HPN). Patient records were reviewed to obtain clinical and demographic information and date of death. Anthropometric measurements and BMI contemporaneous to CT scans were recorded.

Results 64 patients met inclusion criteria (M:F 1:1). 83\% of our cohort had LSMI below previously published thresholds for sarcopenia. Mean pre-HPN LSMI was $36.5 \pm 6.8 \mathrm{~cm}^{2} / \mathrm{m}^{2}$. Mean BMI pre-HPN was $22.1 \pm 4.8 \mathrm{~kg} / \mathrm{m}^{2}$. Both BMI $(\mathrm{p}<0.001)$ and LSMI $(\mathrm{p}=0.003)$ increased post-HPN. A positive correlation was seen between BMI and LSMI pre $(\mathrm{p}<0.001)$ and post-HPN $(\mathrm{p}=0.003)$. No correlation was seen between LSMI and anthropometric measurements pre-HPN $(\mathrm{p}=0.78)$ or post-HPN $(\mathrm{p}=0.96) .11(17 \%)$ patients died during the study period; a low LSMI pre-HPN was not a risk factor for mortality (HR $0.97 \mathrm{p}=0.55$ ).

Conclusions This study is the first to look at sarcopenia and survival using CT defined LSMI in the IF population. $83 \%$ of our cohort had a pre-HPN LSMI below previously published thresholds, yet we found no relationship between lower baseline LSMI and survival. This may reflect the heterogeneity of the prognoses of the IF population, or that parenteral nutrition itself affects survival. 
Our study showed that LSMI and BMI improved following HPN but demonstrated that other anthropometric measurements had poor correlation with LSMI and showed no significant improvement overall after HPN, confirming the known problems of inter-operator and patient variability of these measurements. Whilst we found significant correlation between LSMI and BMI, BMI significantly underestimated the presence and degree of sarcopenia.

We have shown LSMI can provide an objective and reproducible measure of sarcopenia in IF. Future larger studies should be performed to evaluate associations with patient outcomes and utility in clinical decision making.

\section{OWE-021 DESCRIBING THE GUT MICROBIOME AND METABOLOMIC CHANGES IN BILE ACID DIARRHOEA}

${ }^{1}$ Nidhi Sagar*, ${ }^{2}$ Henri Duboc, ${ }^{1}$ Gemma Kay, ${ }^{3}$ Konstantinos Gerasimidis, ${ }^{3}$ Vaios Svolos, ${ }^{1}$ Alfian Wicaksono, ${ }^{1}$ Christopher Quince, ${ }^{1}$ James Covington, ${ }^{4}$ Nicola O'Connell, ${ }^{4}$ Subiatu Wurie, ${ }^{1,4}$ Ramesh Arasaradnam. 'University of Warwick, UK; ${ }^{2}$ Paris VI University, France; ${ }^{3}$ University of Glasgow, UK; ${ }^{4}$ University Hospitals Coventry and Warwickshire, UK

\subsection{6/gutjnl-2018-BSGAbstracts.317}

Introduction The diagnosis of bile acid diarrhoea (BAD) is often missed or misdiagnosed for irritable bowel syndrome diarrhoea predominant (IBS-D) as these conditions have a similar clinical presentation. The principal hindrance to diagnosis of BAD is limited access to the diagnostic SeHCAT scan. Mechanisms of aetiology underlying BAD have not been fully elucidated but it has been accepted that bile acids (BAs) are metabolised by the gut microbiota, therefore their role as signalling molecules in regulating intestinal homeostasis is influenced primarily by the gut commensals.

The aim was to profile the gut microbiome in BAD and investigate the mechanisms of how bacterial metabolic products may influence the development of disease.

Methods 157 patients participated in the study after having a SeHCAT scan to either diagnose BAD or IBS-D (the latter with a negative scan). Exclusion criteria included recent use of antibiotics/probiotics and the diagnoses of coeliac disease, colorectal cancer and active inflammatory bowel disease (unremarkable levels of C-reactive protein and/or faecal calprotectin were required) in type $1 \mathrm{BAD}$ patients. To examine the gut microbiome, 16S ribosomal RNA gene analysis was undertaken. Bacterial metabolites (short chain fatty acids-SCFAs and volatile organic compounds-VOCs) and BAs were measured using gas and liquid chromatography, mass and ion mobility spectrometry.

Results Intestinal dysbiosis with reduced bacterial diversity was observed in patients with BAD compared with IBS-D $(p=0.01)$. A greater total concentration of SCFAs $(p=0.17)$ with increases in the concentrations of acetate $(p=0.14)$ and propionate $(p=0.08)$ were observed in BAD compared to IBSD. An increase in the concentrations of faecal primary BAs $(p=0.05)$ and serum CDCA $(p=0.05)$ was observed in BAD compared to IBS-D.

Conclusions Intestinal dysbiosis with altered fermentation and resultant BA dysmetabolism were observed in BAD. The metabolic output of the microbiota rather than abundance of specific bacterial taxa appears to be more important in the aetiology of BAD.

\section{OWE-022 GLUTEN FREE DIET ADHERENCE ASSESSMENT USING CDAT AND BIAGI QUESTIONNAIRES IN PATIENTS WITH COELIAC DISEASE}

Michelle S Lau*, Peter D Mooney, Michael A Rees, William L White, Lauren J Marks, Marios Hadjivassiliou, David S Sanders. Academic Unit of Gastroenterology, Royal Hallamshire Hospital, Sheffield, UK

\subsection{6/gutjnl-2018-BSGAbstracts.318}

Introduction Non-adherence to a gluten free diet (GFD) could lead to persistent villous atrophy and the development of complications in coeliac disease. However, current adherence assessment methods have their own limitations: dietetic evaluation by a specialist dietitian is usually accurate but resources are often limited; serology has been shown to have poor sensitivities in measuring adherence; and although duodenal biopsies are considered the gold standard, it is invasive and expensive. We aimed to assess the sensitivities of 2 validated questionnaires, which have been suggested as non-invasive markers of adherence.

Methods We prospectively recruited coeliac disease patients who were referred to secondary care for further evaluation of dietary adherence and disease remission. All patients were tested with tissue transglutaminase (IgA-TTG) and endomysial antibodies (IgA-EMA), and completed 2 adherence questionnaires, one devised by Biagi et al., ${ }^{1}$ and Coeliac Disease Adherence Test (CDAT) devised by Leffler et al. ${ }^{2}$ The questions were largely related to the patients' attitude and strategy towards gluten avoidance. The Biagi questionnaire consists of 4 questions, and the score ranges from $0-4$; score $<3$ indicates non-adherence. CDAT consists of 7 questions, and the score ranges from 7-35; score $>13$ indicates non-adherence. Duodenal biopsies were taken from all patients, and the presence/absence of villous atrophy was the reference standard used to determine the sensitivities.

Results A total of 120 patients were recruited, 80 females (67\%), median age 54, median duration of GFD 5 years. Forty-five patients $(37.5 \%)$ had persistent villous atrophy. The median CDAT score was 12 , and the median Biagi score was 3. The sensitivities of the surrogate markers for adherence compared to duodenal histology are demonstrated below.

\begin{tabular}{|c|c|c|c|c|}
\hline & $\begin{array}{l}\text { Sensitivity } \\
\%(\mathrm{Cl})\end{array}$ & $\begin{array}{l}\text { Specificity } \\
\%(\mathrm{Cl})\end{array}$ & $\begin{array}{l}\text { Positive predictive } \\
\text { value } \%(\mathrm{Cl})\end{array}$ & $\begin{array}{l}\text { Negative predictive } \\
\text { value } \%(\mathrm{Cl})\end{array}$ \\
\hline CDAT & $\begin{array}{l}55.4(40.0- \\
70.4)\end{array}$ & $\begin{array}{l}57.3(45.4- \\
68.6)\end{array}$ & $43.9(35.0-53.1)$ & $68.3(59.5-75.9)$ \\
\hline Biagi & $\begin{array}{l}24.4(12.9- \\
39.6)\end{array}$ & $\begin{array}{l}92.0(83.4- \\
97.0)\end{array}$ & $64.7(42.1-82.2)$ & $70.0(62.9-70.8)$ \\
\hline $\begin{array}{l}\text { CDAT } \\
+ \text { +Biagi }\end{array}$ & $\begin{array}{l}62.2(46.5- \\
76.2)\end{array}$ & $\begin{array}{l}56.0(44.1- \\
67.5)\end{array}$ & $45.9(37.6-54.4)$ & $71.2(61.8-79.1)$ \\
\hline IgA-TTG & $\begin{array}{l}40.0(25.7- \\
55.7)\end{array}$ & $\begin{array}{l}97.5(90.7- \\
99.7)\end{array}$ & $90.0(68.7-97.4)$ & $73.0(70.0-77.5)$ \\
\hline $\begin{array}{l}\text { IgA- } \\
\text { EMA }\end{array}$ & $\begin{array}{l}37.8(23.8- \\
53.5)\end{array}$ & $\begin{array}{l}97.3(90.7- \\
99.7)\end{array}$ & $89.5(67.3-97.2)$ & $72.3(67.4-76.7)$ \\
\hline
\end{tabular}

Conclusions CDAT alone was not superior compared to IgATTG $(p=0.6961)$. However, the combination of CDAT and Biagi questionnaires significantly outperformed IgA-TTG $(p=0.0162)$ in detecting villous atrophy. These questionnaires 